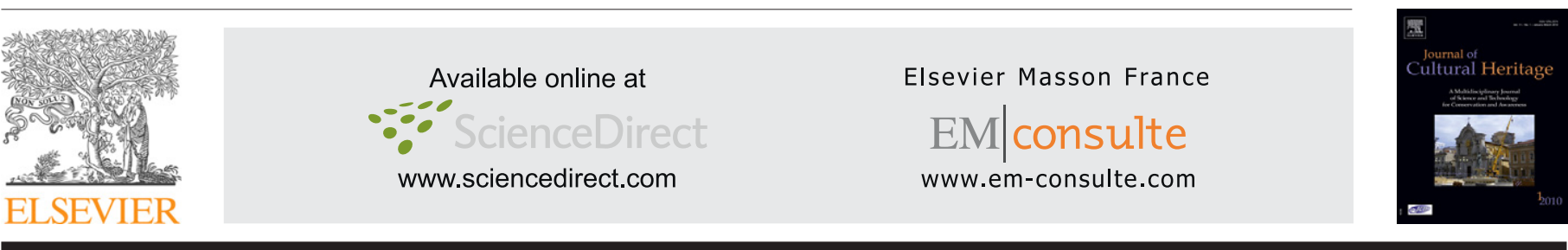

Original article

\title{
ISEE: Information access through the navigation of a 3D interactive environment
}

\author{
Laura Pecchioli ${ }^{\mathrm{a}, *}$, Marcello Carrozzino ${ }^{\mathrm{b}, 1}$, Fawzi Mohamed ${ }^{\mathrm{c}, 2}$, \\ Massimo Bergamasco ${ }^{\mathrm{b}, 3}$, Thomas H. Kolbe ${ }^{\mathrm{a}, 4}$ \\ a Technische Universität Berlin, Institut für Geodäsie und Geoinformationstechnik, Strasse des 17. Juni 135, 10623 Berlin, Germany \\ b PERCRO, Scuola Superiore Sant'Anna, Piazza Martiri della Libertà, 33, 56127 Pisa, Italy \\ ${ }^{\mathrm{c}}$ Humboldt Universität, Institut für Chemie, Unter den Linden 6, 10099 Berlin, Germany
}

\section{A R T I C L E I N F O}

\section{Article history:}

Received 30 June 2009

Accepted 26 November 2010

Available online xxx

\section{Keywords:}

Interactive 3D interface

Virtual reality

Augmented reality

Normalized 3D Gaussian

Spatial relevance

Cultural heritage data

\begin{abstract}
A B S T R A C T
Managing heterogeneous information related to Cultural Heritage sites and artifacts is still a complex task. In latest years, there has been a significant trend towards the massive digitization of this data, as this allows more efficient and reliable storage and management processes. Furthermore, the relationship between conservation managers, who are often unfamiliar with current documentation techniques, and information providers, who tend to be highly technical practitioners without expertise in cultural heritage, is not easy to handle. Moreover, in Cultural Heritage objects often have a strong 3D component, and cannot be easily represented with conventional data management frameworks like Geographic Information System (GIS). The use of a 3D framework may allow a closer adherence to the real world, as it respects the spatial relationships among various parts. A novel method to access spatial information through the interactive navigation of a synthetic 3D model, reproducing the main features of a corresponding real environment, is proposed in this paper. The result of this work is a system called ISEE. An innovative aspect of the ISEE approach is represented by our definition of spatial relevance of information. The information is ranked with a novel measure of relevance that depends on the position/orientation in the 3D space, and allows for an intuitive interface. The basic idea of ISEE is to allow retrieving information by just looking around in a 3D environment, as moving and looking at the world is the main modality we use to gather information from it. Users explore in intuitive way a 3D environment and access the related information, kept in its spatial context. Information are accessed through "extended zones", i.e. portions of the 3D environment not having direct reference to specific elements, rather to the distribution of information and to the current user location. The use of extended zones gives to the proposed ranking algorithm a superior performance than rankings methods based on distance. Indeed the ISEE ranking matches the intuitive expectation of the users, as was verified with a formal usability test. The system has been applied to case studies related both to outdoor and indoor environments, showing its potential also as a smart guide with the use of augmented reality technologies. In order to enable access to a larger audience, sample applications using this method are based on Web technologies and do not require special training to be used. At the end of the paper are presented the results of an evaluation test, which provided useful suggestion to improve the system usability and performances.
\end{abstract}

(c) 2010 Elsevier Masson SAS. All rights reserved.

\section{Research aims}

The goal of this research is to develop a method to store and access cultural heritage related information. Authoring and

\footnotetext{
* Corresponding author. Tel.: +49 3031422 699; fax: +49 3031421973.

E-mail addresses: laura.pecchioli@gmx.de(L.Pecchioli),m.carrozzino@sssup.it.it (M. Carrozzino), fawzi@gmx.ch (F. Mohamed), bergamasco@sssup.it (M. Bergamasco), thomas.kolbe@tu-berlin.de (T.H. Kolbe).

${ }^{1}$ Researcher. Tel: +390583 432 6714; fax: +39 05834326565.

${ }^{2}$ Researcher. Tel.: +49 3020937 140; fax: +49 3020937136.

${ }^{3}$ Full professor. Tel.: +390508 831 11; fax: +39050 883225 .

${ }^{4}$ Full professor. Tel.: +49 3031423 205; fax: +49 3031421973.
}

retrieval of information should be made as simple as possible in order to be used by professionals from different fields and by the general public. Very often this kind of information have a strong 3D component and cannot be easily handled by means of conventional management systems. In order to comply with this requirement and to enhance the user involvement, we propose a method based on the navigation of an interactive 3D environment. The design of a 3D interactive interface [1] suitable for such a purpose is not a trivial task. Current selection methods, even the most established ones, have several drawbacks (tricky management of hidden or overlapping objects, sensitivity to the distance, etc.). In order to overcome these issues and to keep the navigation simple, the system should allow an intuitive interaction, where information 
is accessed by simply looking at the environment where it is contextualized, as this is the solution closest to the normal human behavior. It is also needed a way to efficiently filter information, which occasionally may overwhelm users if a high-density data sector is under observation, therefore a ranking system based on relevance is strongly advisable.

Such a framework is the ideal environment to enable knowledge exchange among users. It has all the characteristics to be an advanced authoring environment, where the main actor is the user.

Finally, the implementation of the system should run on standard Web browsers, in order to allow access to the widest audience without any special requirements.

\section{Introduction}

The whole field of preserving, documenting and studying Cultural Heritage is an interdisciplinary field that draws together several different competencies. Information is gained from different sources and in various formats. Furthermore, the relationship between conservation managers, who are often unfamiliar with current documentation techniques, and information providers, who tend to be highly technical practitioners without expertise in Cultural Heritage, is not easy to handle.

This system targets two specific segments of public interested or involved in Cultural Heritage: general public and professionals. In order to keep into account the different needs of these segments, the approach must provide also a higher access level to professionals, which would manage and manipulate information with tools specific to their field, insert more detailed data, and decide presentation and retrieval modes. Ideally, the proposed approach should be able to provide the basis to create a community for the common language, where the people can understand, query, learn and leave opinions or sharing useful information.

There are many kinds of textual, geometric or geographical information, images (orthophotos, normal photos and planes), or $3 \mathrm{D}$ information, which exist in very different formats. Sources can be historical documents or the result of recent analyses. In latest years, there is a significant trend towards the massive digitization of this data, as this allows more efficient and reliable storage and management processes. A significant part of digital information related to Cultural Heritage sites and artifacts is based on pre-existing works that have been subsequently digitally reproduced. However, nowadays, new information is frequently produced directly in a digital format, and very often it exists only in this form.

Unfortunately, having the information in a digital format is not enough. This raises non-trivial issues on the long-term preservation of this data, along with issues on their future understandability and handling. Europeana ${ }^{5}$ represents an example of an actual large project that tries to tackle these issues.

The application of virtual technologies in Cultural Heritage has proved to effectively help the understanding and the visualization of reconstructions of monuments, sites and settlements. In particular, novel interaction paradigms have been produced in the field of architectural conservation, resulting in a promising reference point for the conservation and the maintenance of historical buildings in the future [2]. However, although current virtual technologies and methodologies allow for the production of 3D results effectively used for many purposes, such as archaeological documentation, digital conservation, support to restoration, the ability to easily interact with a large $3 \mathrm{D}$ model is still a relevant issue.

\footnotetext{
${ }^{5}$ Europeana functions like a multimedia Internet portal with content from different sources (in literature, history, art or cinema a simple route to access European cultural resources). This important and big project offers a simple way to find cultural material from across Europe in digitized format (http://www.europeana.eu).
}

Indeed, high-quality 3D models still need a huge amount of data in terms of geometrical details and textures, and this very often limits the possibilities of their interactive real-time visualization. In [3] current surveying and 3D modeling methodologies are thoroughly reviewed exposing their limitations, issues and potentialities in the cultural heritage field. Despite a number of weaknesses, reported examples show the potential of 3D technologies to digitally document and preserve our heritages as well as providing a means for sharing and managing.

In the latest years many software applications have been developed to visualize, archive and share 3D documentation and conservation data, although a traditional approach has often been used due to the complexity of its interdisciplinary relationships. Novel approaches try to address the increasing need for automated 3D modeling in order to assist the production of 3D data, usually requiring huge efforts in terms of manual work and human input [4].

However, there is a number of key questions which still do not present a clear and well recognized answer:

- how to provide a unified methodology to information management, often deeply inhomogeneous and non-uniform, like that involved in Cultural Heritage ICT applications?

- how to provide an efficient way to deal with big amounts of information often contained in a small physical space (high density of information)?

- how to enable access to this kind of information to large audiences of professional and non-professional users?

- how to make this audience an active part in the process of information management?

Our objective is to provide an answer to these questions, keeping into account the specificity of the context (Cultural Heritage related ICT applications) and, at the same time, aiming to establish a technology-enhanced methodology, which can be easily adapted to other similar contexts.

Our research focus in particular on the restoration field, where several types of documents and formats are present and, often, the only unifying entity is the object, which the information is related to. Furthermore, in this case 3D information assumes a particular relevance since the involved objects cannot be so easily reduced to a $2 \mathrm{D}$ representation without loss of significance.

Preservation of the digital heritage requires sustained efforts on the part of governments, relevant industries and heritage institutions. The understanding of the limitations and potentialities as well the visualization issues, involved in the cultural heritage field could reinforce international cooperation and solidarity to enable all countries to ensure dissemination and continued accessibility of their digital heritage (UNESCO Charter on the Preservation of the Digital Heritage, 2003). In [3] some examples of UNESCO world heritage sites 3D documentation are reported and discussed. Undoubtedly our heritage would benefit of more international collaborations and information sharing to digitally preserve and make them more widely accessible in different formats and to as many users as possible users. Web applications in Cultural Heritage are increasingly used because they allow access from any computer, while keeping a centralised repository of information. The quality of their interface has practically reached parity with desktop applications. The prototypes developed in this work are web applications that allow a person to keep the information in its context. This aspect is very important especially with monuments, in archaeological sites, or in a museum, to be able easier to access information and in real time can help to understand it better.

Although many of the projects so far introduced have tried, in an integrated fashion or partially, to setup a methodology to assist visualizing and querying archival resources, to improve informa- 
tion sharing and knowledge creation, and to provide easier access to information, there is currently no software that is fully able to cope with the complex needs of cultural assets management; the language to be used to deal with information in 3D is still very much an open research problem.

The use of Virtual Reality can help to understand and to manage the real world, but the transition between these realities is not easy and the result does not always satisfy expectations. VR reconstructions can be appealing for general public, but for specialists this approach might sometimes be not necessarily so useful and can even be misleading. In our work, an important set goal has been to create an interactive 3D geovirtual environment where it is possible to access information while navigating within the model; therefore, the added value of 3D lies not only in the appeal of the representation but also in the means it provides to read content.

\section{The concept}

We started considering what are the requirements for an adaptive and intuitive interface to access information connected with a spatial region, but not reducible to simple numerical data (texts, tables...), i.e. what in GIS is called geo-coded information. This information is naturally embedded in $3 \mathrm{D}$ spatial contexts, and the most natural way of behaving in such a context is to just look around; indeed, seeing is an essential action common to humans and in our natural behavior since birth. By using sight, we naturally focus on something; therefore, we propose to use this action as a common language, which people can use to understand, query and insert information. To achieve this, it is useful to define the region of the 3D space which the user is focusing at a certain moment. We call this region the View Zone (VZ). The user can then be interested either in extracting relevant information or in adding new information related to that zone. This is the core of ISEE (which is clearly named after this evidence): using sight to actively query and insert information.

This realizes one of the most important goals in our approach: to ease everybody's access to information. The complexity dictated by the type of data is simplified in a few easy moves (navigating an environment and looking around); moreover, we think that this involves also an edutainment component which helps users in getting more involved in the experience.

To implement this idea, it is necessary to link each piece of information with a region of the space, find a precise definition of the $\mathrm{VZ}$ and define a ranking to find the relevant information. As this ranking can only depend on the VZ and the location of the information, i.e. on spatial relationships, we call it spatial relevance.

\section{The information zone (IZ)}

In ISEE, information is associated with regions of the 3D space, which we call IZ. This differs from other existing systems where information is often associated with single points or 2D areas [1,5]. IZs do not necessarily coincide with a 3D object represented in the model, they might be just a part of it, or they may include many objects at the same time.

This kind of information association has a clear advantage. It is not necessary to subdivide 3D models into logical sub-elements to attach information. So one can directly use meshes generated by 3D scanners (or any automatically generated 3D model), without needing any particular post-processing in order to identify subportions of the models.

\subsection{Gaussian function}

The definition of the $3 \mathrm{D}$ zone which information are linked to is crucial. Our first choice was to use spheres, as they are the simplest 3D objects and quite easy to define interactively (just two parameters are needed, radius and centre). However a sphere, like any other solid object, has a sharp boundary: a point is either inside the sphere or outside it. In order to avoid this binary behavior, which may lead to lose relevant information close to the boundaries, a 3D zone, which does not coincide exactly with a definite volume and has a smooth transition at its boundaries would be preferable. This can be achieved by associating a quantity, that we will call concentration of information, to each point of the space, representing how much information is contained at that point in the zone. We decided to use 3D Gaussians to define this concentration [6].

In fact, a 3D Gaussian is a function which assigns a value to each point of the space (it can be seen as a fog, more dense in the centre, and less dense on the periphery), which can be used to adequately describe the concentration of information.

The IZ is therefore realized by associating information with a 3D Gaussian centered on a relevant point of the object which we want to link the information to, and with a width that should be comparable to the size of the object/detail the information refers to. It is important to note that the Gaussian is not used to define the exact information location, but rather an approximated zone, which can be subsequently used to search and retrieve the information. The amount of information is not connected with the size of the Gaussian, which depends just on the size of the object. First of all, all Gaussians should represent the same amount of information regardless of their size. The use of a continuous distribution nicely solves this problem: Gaussians are normalized and, while a sharp Gaussian is smaller than a loose one, it also has a higher concentration so that the total amount of information is the same (Fig. 1).

In the current stage of development, a more precise definition of the zone can be stored in the information data itself. In the future, one might directly support more complex zones.

\subsection{The $V Z$}

The portion of the 3D model which is being looked at in the interactive 3D Viewer is also approximated with a normalized 3D

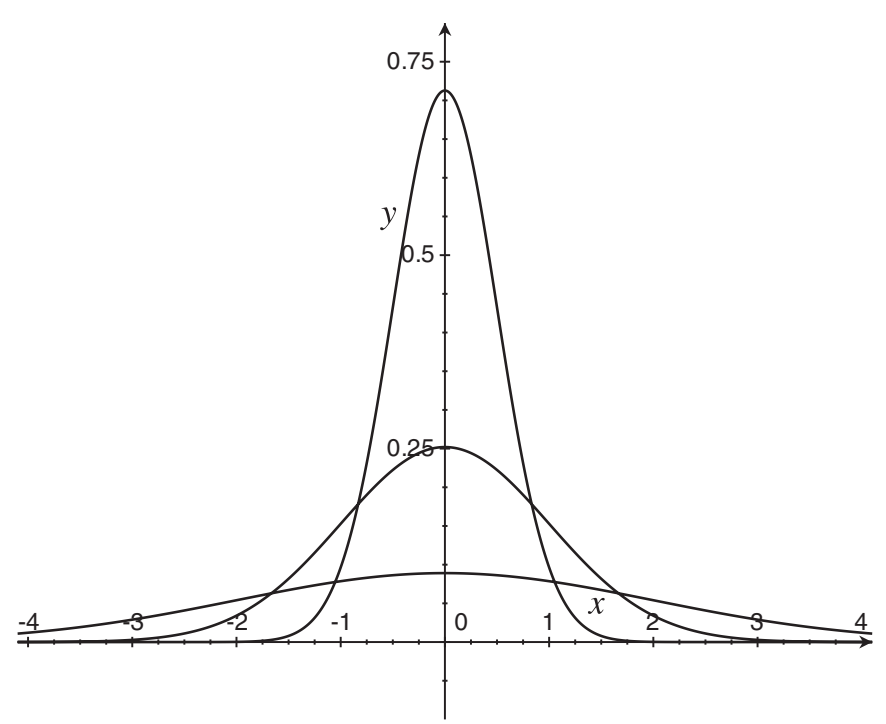

Fig. 1. Plot of normalized gaussians with width $=0.5,1.0,2.0$ as function of the distance from the centre. 


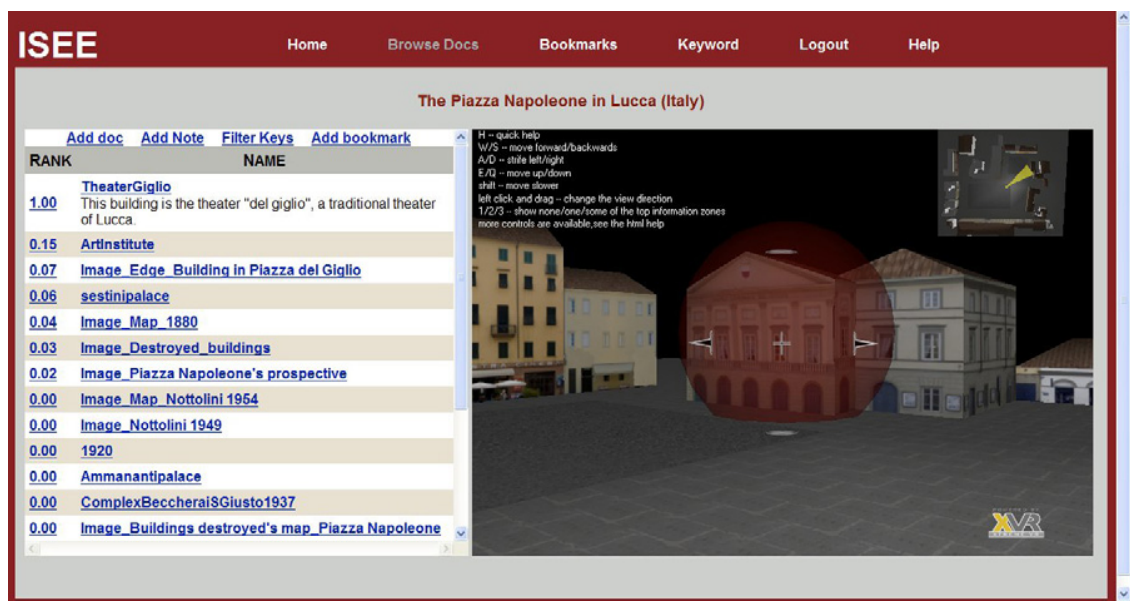

Fig. 2. Interface of ISEE showing the View Zone as cross-hair and Information Zone as a sphere.

Gaussian, so that VZs and IZs are described in a similar way. The symmetric treatment of VZs and IZs allow us to use the interactive 3D viewer to visually insert the $\mathrm{IZ}$ of a piece of information (authoring), or to jump immediately to the view related to some information (retrieval).

The Gaussian that represents the VZ is built as follows:

- the centre of the Gaussian is chosen on the point P, representing the first intersection between the 3D scene and a ray cast from the current point of view towards the centre of the view plane;

- the width of the Gaussian is chosen proportional to the distance of the centre $P$ from the view point, so that it is similar to the size of the viewed area.

The of the view cone is constant, and the closer the object is, the smaller the width, so that the perceived dimension of the VZ on the screen is constant, but its real size depends on the distance from the object (Fig. 2).

As a matter of fact, this implements a sort of automatic level of the detail of information, which can be used to filter high-density information spaces and reduce the amount of retrieved data.

From this point of view, ISEE method represents an improvement on the insufficient filtering performance of distance-only methods, which typically use the distance from the user as an extra simplification (e.g., Google Earth $5^{6}$, Microsoft Virtual Earth ${ }^{7}$ ). Indeed, their lack of performance is not only an academic problem; map based systems often introduce discrete levels of detail, depending on the zoom level, in order to avoid overwhelming the user with too much information.

In an interactive visualization of a 3D model the scale depends on the distance of the object looked at, therefore in the same scene the same object can have infinite levels of detail. It is clear that a continuous approach that does not arbitrarily classify information in a given predefined set of discrete levels of detail is advantageous.

Our method extends the ray-casting selection method in 3D environments, which typically allows selecting objects/regions/points with a simple binary behavior: either the selected point is associated to some information or it is not [7]. Moreover with them there is no straightforward solution to select hidden or obstructed objects using this method. In the next section we will show how the determination of an extended VZ, rather than a simple point/object selection, can be valuably

\footnotetext{
${ }^{6}$ http://www.google.com/earth/index.html.

7 http://www.microsoft.com/maps/.
}

exploited to retrieve information and have a direct estimate of its relevance.

\section{Relevance}

An innovative aspect of this approach is represented by our definition of the spatial relevance of information.

What we want to develop is a continuous measure of the relevance depending on the spatial relationships between VZ and IZ Intuitively, the relevance of information should be maximal when its IZ coincides with the Viewed Zone (VZ), decreasing when they are far apart.

When browsing the data using this measure of relevance, an ordered list of the most relevant information of the selected area (Data List) related to the VZ is built.

To implement this ranking we need a precise and computationally efficient definition of spatial relevance. We can valuably use the overlap between the Gaussians VZ and IZ as a measure of the relevance of the information connected to the current view. This allows us to associate a continuous level of detail to the information.

Given two normalized Gaussians:

$g_{A, B}(r)=\frac{1}{\left(2 \pi \sigma^{2}\right)^{3 / 4}} \exp \left(-\frac{\left|r-R_{A, B}\right|^{2}}{2 \sigma^{2}}\right)$

the overlap between them is defined as:

$S_{A B}=\int g_{A}(r) g_{B}(r) d r=\left(\frac{2 \sigma_{A} \sigma_{B}}{\sigma^{2}+\sigma^{2}}\right)^{3 / 2} \exp \left(-\frac{\left|R_{A}-R_{B}\right|^{2}}{2 \sigma^{2}+\sigma^{2}}\right)$

The overlap $\mathrm{S}_{\mathrm{AB}}$ is maximal when the two Gaussians coincide $\left(R_{A}=R_{B}\right.$ and $\left.\sigma_{A}=\sigma_{B}\right)$ and gets smaller if the IZ is far away or very differently sized (Fig. 3).

The choice of using 3D Gaussians allows to keep the method simple and efficient (other functions would probably need a numerical integration in 3D to evaluate the overlap). Although this choice is not the best for precise queries, it works perfectly in facilitating fast approximate queries that can be subsequently refined interactively by the user with more traditional means, like keyword filtering.

\section{Case studies}

The prototypes developed in this work are web applications, making use of the XVR technology [8], where the user can interactively navigate a 3D model representing a cultural site and retrieve linked information. The implemented case studies are related both to outdoor and indoor environments: 


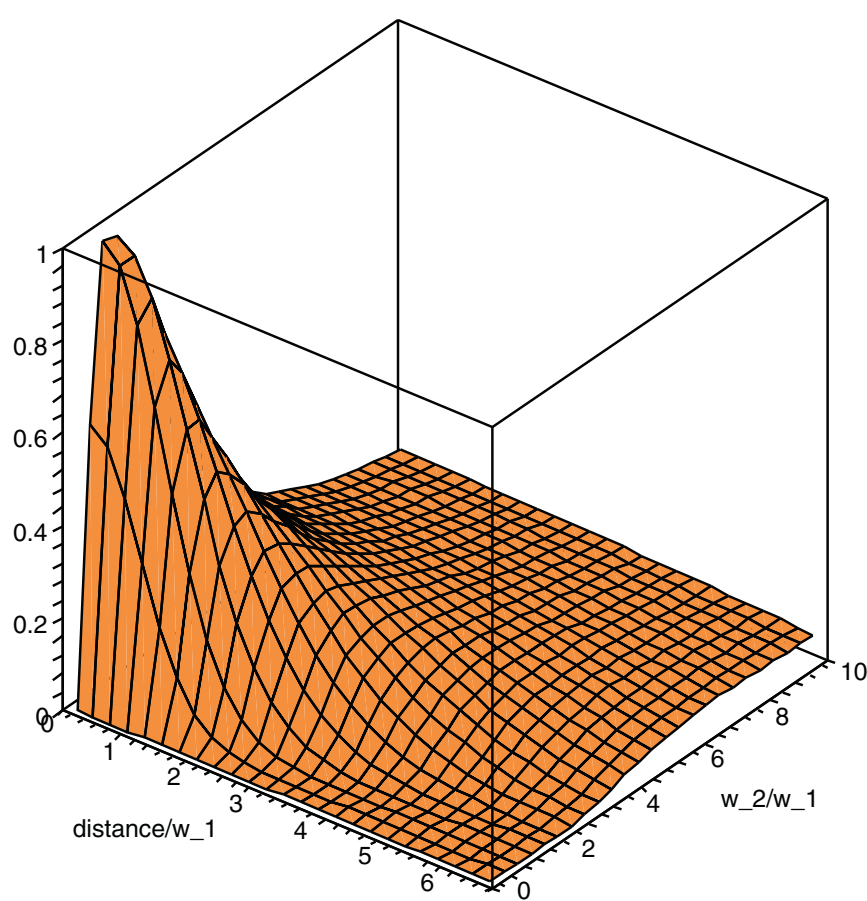

Fig. 3. $3 \mathrm{D}$ plot of the overlap between two normalized Gaussians of width w 1 and w2.

- Piazza Napoleone in Lucca (Italy, outdoor);

- the crypt of the Cathedral of St. Servatii in Quedlinburg, SaxonyAnhalt (Germany, indoor);

- MuS's (a 3D virtual indoor environment).

The 3D models used in these case studies are synthetic geovirtual environments produced using different source data. The model of the Piazza Napoleone was manually created starting from a topographic geo-referenced map with the real height of the buildings. The database has been subsequently built with documents found in archives, libraries and personal photos. The information inserted in the database is related to the urban changes and the artistic/architectural aspects of the square (Fig. 4).

The second case study deals with Quedlinburg, a village in Sachsen Anhalt. The number and high quality of the timber-framed buildings make it an exceptional example of a medieval European town and it is part of the World Heritage List (UNESCO). The cathedral of St. Servatii has a crypt with paintings that represent one of the largest painting cycles from 12th century in Germany, covering

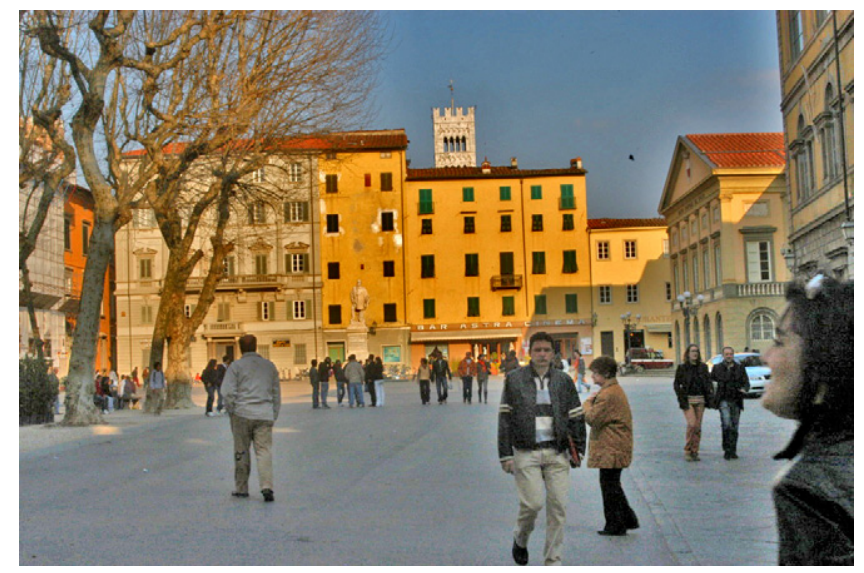

Fig. 4. Piazza Napoleone in Lucca.

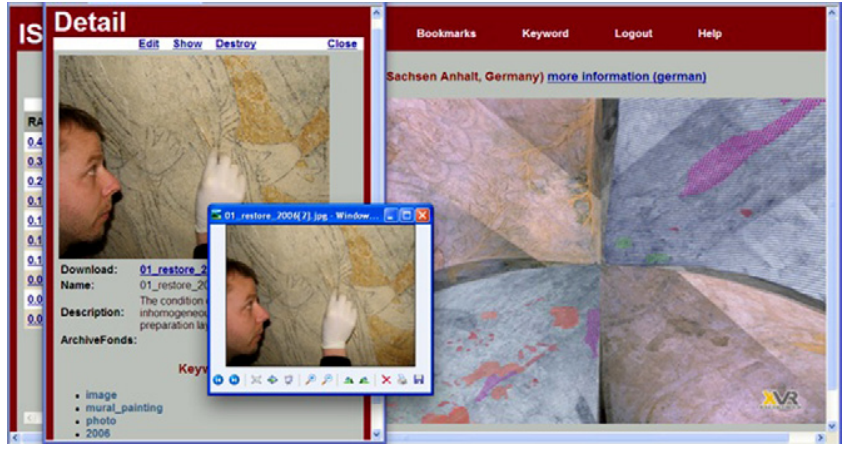

Fig. 5. The crypt in St. Servatii in Quedlinburg with the mapping of the state of conservation.

an area of more than $120 \mathrm{~m}^{2}$. They were for a long time covered, and only in the beginning of the 20th century, were they discovered again $[9,10]$. The model of the crypt has been realized from an existing geo-referenced cloud of points obtained by a 3D scanner. Issues were mainly related to the complexity of the model, which had to be reduced in order for it to be suitable for real-time navigation, and to the creation of an appropriate 3D interpolated shape so as that the data already available could be easily imported. Data is related to the conservational work of the painting ceilings conducted from 2000 to $2007^{8}$ in the form of maps, orthophotos and data from archives [11]. Orthophotos have been transformed into textures, and so it was possible to transfer the GIS data connected to them to the 3D model and vice versa (Fig. 5).

In the case of the 3D virtual museum called MuS's, we have applied our method to a more complex topology. The user can freely explore museum accessing information by looking at a picture or an object (Fig. 6).

In addition, in the case study of Piazza Napoleone, we tested a real world outdoor application using a GPS Compass (Vector CSI Wireless), providing 2D heading and positioning data and connected to a laptop (Pentium IBM ThinkPad T42) running the ISEE system, that allowed us, while walking in the real square, to access the database by simply looking at the elements from which we wanted to retrieve information [12]. The test proved that the system is able, exactly like in digital environments, to provide in real-time relevant information about the elements of the real environment currently observed and suggested, as the next step, to implement it as an Augmented reality smart guide (Fig. 7).

\section{Evaluation}

The system has been evaluated through a usability test on eight people: four women and four men between the ages of 28 and 38, coming from different backgrounds: computer scientists, archaeologists, researchers, pediatricians, engineers and personal account managers. Users had to follow a pre-ordered task of about 20-25 minutes. After the explanation of the usability test, Users had to follow a pre-ordered task of about 20-25 minutes. After providing a brief overview of the test, users were given two minutes time in order to get familiar with the interaction, without giving any help other than the one given by the system itself (so as to evaluate the friendliness of the interface). After this time users had to navigate to some predetermined places and retrieve related information. They were then asked to add a note about a detail of their choice, and then retrieve again the information they had just

\footnotetext{
${ }^{8}$ All information related to the crypt in St. Servatii in Quedlinburg collected over a period of about six years produced by the students and Prof. Heinz Leitner of the
} Hochschule für Bildende Künst in Dresden, Germany. 


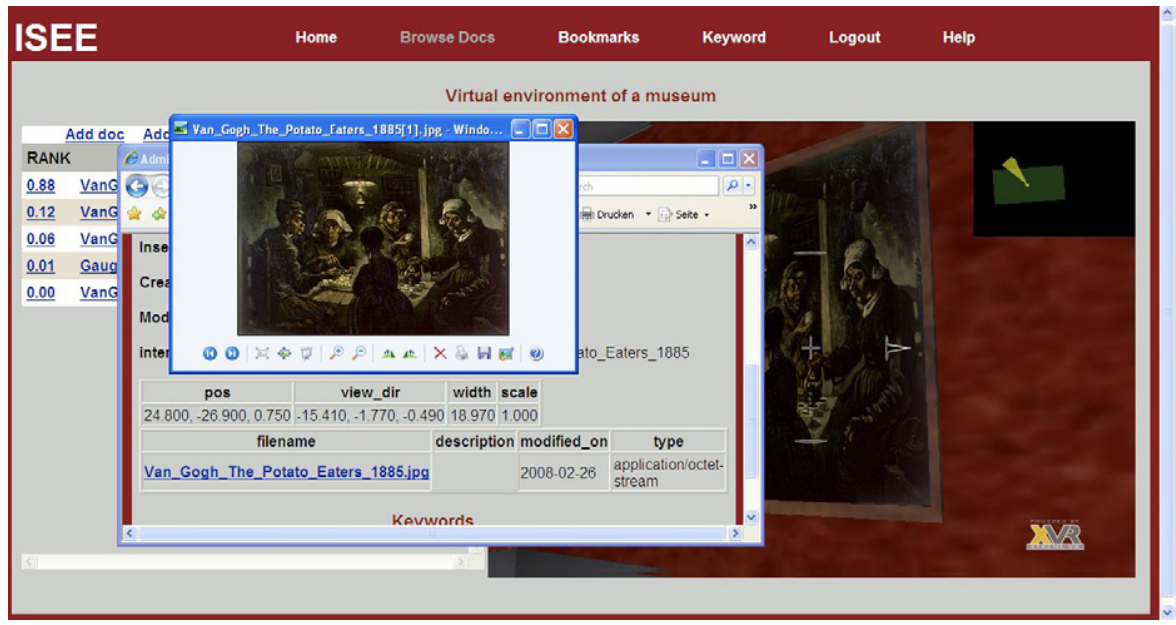

Fig. 6. The virtual museum MuS's.

added after accomplishing other navigation tasks. At this point, if the users hadn't yet discovered a certain functionality of the interface, they were explained about that (for instance, the "fly to" feature, allowing going from an information to its reference in the 3D model, was not easily discoverable but, once explained, it was used very much). At the end of the test, a form with multiple choice and some open questions (mainly about 3D navigation, friendliness of the interface, and relevance of information) was given to users.

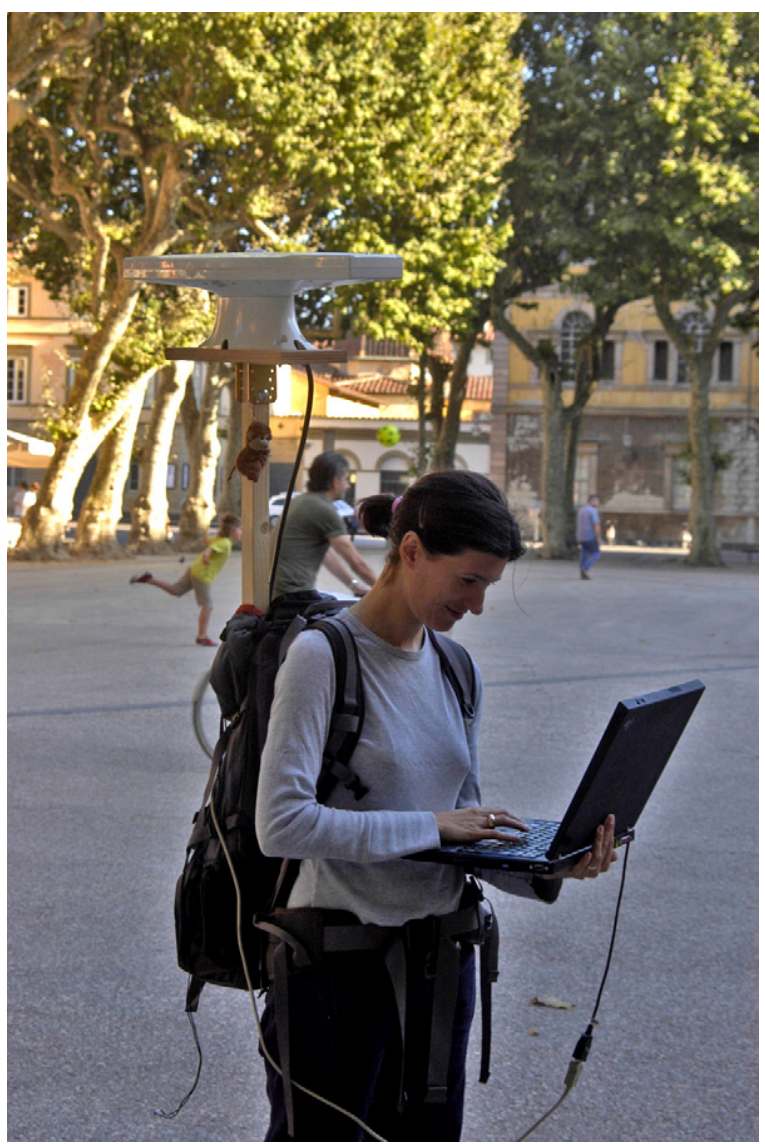

Fig. 7. ISEE live in the real Piazza Napoleone.

\subsection{Usability test}

The test has been carried out on the first prototype dealing with Piazza Napoleone in Lucca. At the end of the test, the user had to answer some questions about his/her familiarity with using a computer and specific questions concerning ISEE. Possible answers where chosen in a 5-point Likert scale, where: 1 = strongly disagree, 2 = disagree, $3=$ neither disagree nor agree, $4=$ agree, $5=$ strongly agree; the final question was open to allow suggestions of any type.

During the test, we also proceeded to the direct observation of some of the users. Users were initially asked to choose the input device for navigating the virtual environment and the related options. This process takes about 3 minutes. The presence of the "quick Help" function proved to be very useful and was used often for the first 5 minutes. The possibility to jump from the information to the model and vice-versa, using links in the ranked list, was deemed not only useful but also very enjoyable. This option helps to have a fast and natural interaction.

After 10 minutes, users tend to become familiar with the method. They also like having the option of downloading data and leaving personal notes.

\subsection{Results analysis}

Almost all users have chosen a combination of mouse and keyboard to navigate the model, with some preference also for the mouse-only option.

The questionnaire was divided into three parts: navigation, information and a few questions about the Website. All users gave comments on the method and suggestions on how to improve it (Figs. 8-10).

The results of the usability test on the whole have been very satisfying, showing that people have found the interaction intuitive, paying attention to the content and quality of the information filtering. The visualization of a $2 \mathrm{D}$ map in the $3 \mathrm{D}$ navigation window proved to be very useful for everybody.

The Add Note function was very popular, as it supports the Web 2.0 trend where users become information providers; using this feature users could easily add personal information to the environment.

In particular the visualisation of the ranked list and the possibility to jump to the view corresponding to a list item was very much appreciated, but had to be previously explained as the users were not able to autonomously figure it out. 


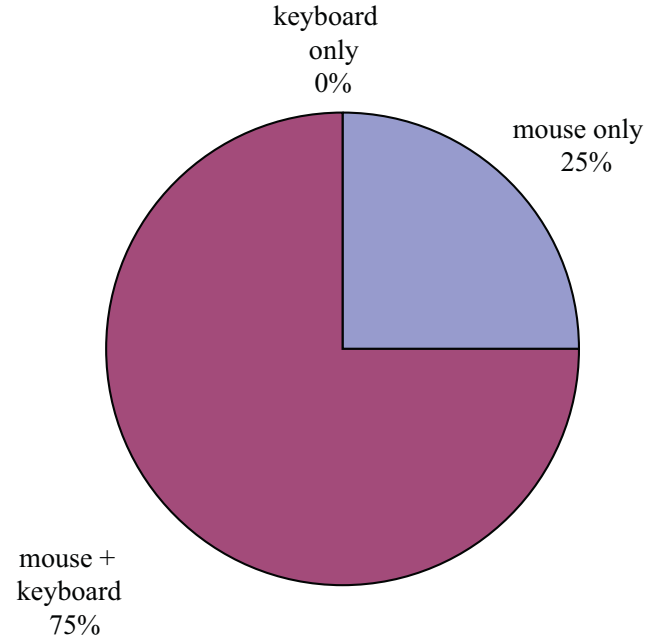

Fig. 8. Graphic on the input devices.

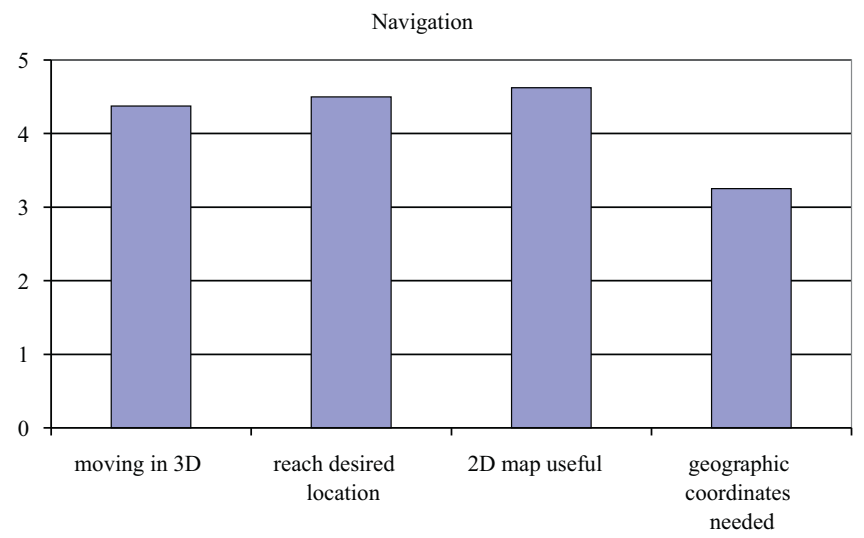

Fig. 9. Results of usability test about the Navigation ( 5 very good/strongly agree, 1 very bad/strongly disagree; see text).

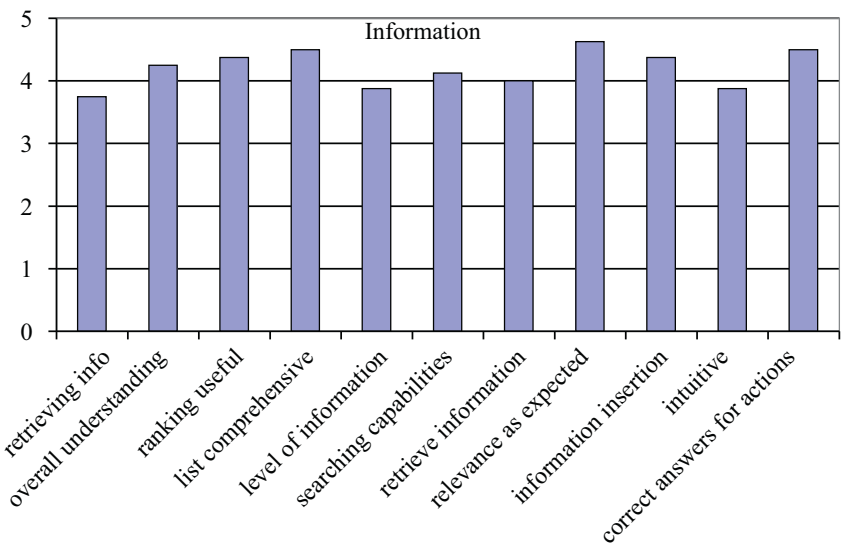

Fig. 10. Results of usability test, Information.

The usability test gave also various suggestions about possible improvements. The method was considered intuitive, but more contextual information could be shown to reduce the Click Distance $^{9}$ to improve the interaction (especially in the informa-

\footnotetext{
${ }^{9}$ Click distance is the number of clicks it takes to get to a web page or document from the homepage of the site. Essentially, the lower the click distance, the more value given to the document.
}

tion Detail window). For instance, users would have liked a bigger preview image in the Detail view.

The users would also have the possibility of uploading documents more easily, inserting less information, and having document types automatically recognized. In one case study, some of the information was connected to destroyed objects; a better way to visualize the presence of "hidden" parts would have been appreciated in this case.

The Help manual was found very useful, but not exhaustive, so it was extended keeping into account users suggestions.

The possibility to directly link the Archive Sources, which was disabled during the test, was considered important. This will help the research, especially when users want to delve further into the information, and represents a connection with the real world (Fig. 11).

The ability of moving around and retrieving information by means of a simple and natural interface was deemed particularly interesting. The choice of manipulation (keyboards and mouse) yielded good results and was easy enough for everybody to use. A common request was to have the possibility of using ISEE in a real museum.

The most common comments were about having more information (especially historical information) in the system. This shows that the system was simple enough to be not an issue so that users could concentrate rather on the content, on its meaning and quality.The detailed scenario descriptions and evaluation results are given in [13].

\section{Conclusions}

We can now look again at the key questions formulated in the introduction. As with all complex questions there is not a single answer, and different approaches might find different answers, that still give a working solution.

Here we try to explain the answers we gave using our approach.

How to provide a unified management methodology to information, often deeply inhomogeneous and non-uniform, like that involved in Cultural Heritage ICT applications?

The ISEE methodology addresses this issue proposing an approach to treat cultural information in a uniform way. Like GIS it references data using spatial information, but it is geared toward 3D data, and based on the use of an intuitive interface.

How to provide an efficient way to deal with big amounts of information often contained in a small physical space (high density of information)?

The ISEE basic idea is to allow retrieving information by just looking around in a 3D environment, as moving and looking at the world is the most natural way we use to gather information from it. As the amount of information we retrieve in this way can be sometimes overwhelming, ISEE shows only the pieces of information relevant for the current view, i.e. data more strictly related to what we are currently looking at.

This approach is possible by calculating a measure of spatial relevance, which depends from the relationship of the location of information (IZ), and the region currently being looking at (VZ). The spatial ranking (achieved calculating the overlap of these normalized regions) mirrors the intuitive expectations: if a user is concentrating on a detail, the related information will have a more significant meaning (a higher rank) than the whole object, whereas if he/she is looking at the whole object, it will result in the opposite behavior.

This ranking method has several advantages over previously used methods, especially with high densities of data and partially overlapping regions. As it uses the size of regions (level of detail) to filter the information, it has a better performance than purely dis- 


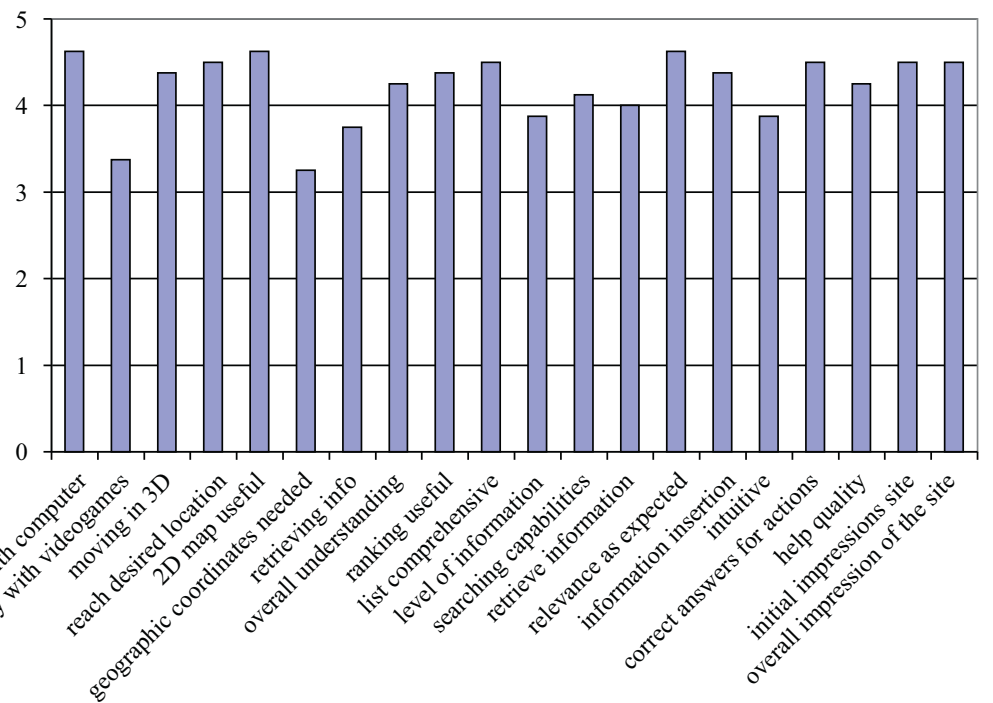

Fig. 11. Summary of the test.

tance based methods. Unlike selection methods like ray casting, it copes well with overlapping and partially hidden regions and avoid users needing to be extremely precise to find information they are interested in. Moreover, a user can interactively refine his search either by carefully focusing on the parts of the model he is looking at, or by more traditional means using keywords based filtering.

How to enable access to this kind of information to large audiences of professional and non-professional users?

The chosen access metaphor, based on natural interaction and exploiting virtual and augmented environments, appears as one of the most appropriate to involve users of different backgrounds and allows them to easily interface with such a system without owning technical skills and without requiring dedicated technological tools.

How to make this audience an active part in the process of information management?

A very interesting side effect is that the same philosophy can be used also for authoring. Information insertion is in fact very simple, as the user can add in every moment specific data about his current view. This opportunity, in addition to the adaptability of the ISEE method to mobile devices, fosters the creation of a community of active users able to deliver information and share experiences.

The system has been applied to selected case studies related both to outdoor and indoor environments, proving potentially to be also an interesting prototype as a smart guide with the use of augmented reality technologies. Currently available mobile devices, such as Iphone and other smart phones, nowadays support the hardware requirements (3D capabilities and positioning/orientation retrieval) to use ISEE for this purpose and it appears natural to port ISEE on these platforms as the very next step of our research.

\section{Acknowledgement}

Part of the research activities dealing with Web3D cultural applications has been carried out in the context of the St@rt regional research project.

\section{References}

[1] D.A. Bowman, E. Krujff, J. La Viola, I. Poupyrev, 3D User Interfaces, in: Theory and Practice, Addison-Wesly, USA, 2004.

[2] C. Chen, M. Hsu, Y. Lee, K. Sun, Y. Lin, W. Lee, Applying Augmented Reality to visualize the history of traditional architecture in Taiwan, in: Proceedings of XXII CIPA Symposium, 11-15 October, Kyoto, Japan, 2009.

[3] F. Remondino, A. Rizzi, Reality-based 3D documentation of World Heritage Sites: methodologies, problems and examples, in: Proceedings of XXII CIPA Symposium, 11-15 October, Kyoto, Japan, 2009.

[4] S. Nitin Jain, S.S. Rohini, S.M. Sabarish, S. Srinath Sridhar, R. Vidhya, Documentation of architectural scenes using a hierarchical method, in: Proceedings of International Archives of Photogrammetry, Remote Sensing and Spatial Information Sciences, Vol. XXXVIII, Part 5 Commission V Symposium, Newcastle upon Tyne, UK, 2010.

[5] C. Andujar, F. Argelaguet, Friction surfaces: scaled ray-casting manipulation for interacting with 2D GUIs, in: R. Hubbold, M. Lin (Eds.), Proceedings of Eurographics Symposium on Virtual Environments, Lisbon, Portugal, 2006.

[6] L. Pecchioli, M. Carrozzino, F. Mohamed, H. Leitner, Accessing information through a 3D interactive environment, in: J. Trant, D. Bearman (Eds.), Proceedings of ICHIM07, International Cultural Heritage Informatics Meeting, Toronto, Canada, 24-26 October, 2007.

[7] M.R., Mine, ISAAC: A virtual environment tool for the interactive construction of virtual worlds. Technical Report TR95-020, UNC Chapel Hill Computer Science, 1995.

[8] M. Carrozzino, F. Tecchia, S. Bacinelli, M. Bergamasco, Lowering the Development Time of Multimodal Interactive Application: the Reallife Experience of the XVR project, in: Proceedings of ACM SIGCHI International Conference ACE, 19-22 November, Valencia, Spain, 2005, pp. 270-273.

[9] F.Gosslau, R. Radecke, Die Stiftskirche zu Quedlinburg, Eine Fürung durch den romanischen Sakralbau und den Domschatz, (Eds.) Convent-Verlag, 1992, Quedlinburg.

[10] Voigtländer, Klaus, Die Stiftskirche zu Quedlinburg - Geschichte ihrer Restaurierung und Ausstattung, Akademie Verlag, Berlin, 1989.

[11] H. Leitner, La conservazione delle pitture murali nella Cripta di San Servatii a Quedlinburg, in: A. Ricerche (Ed.), Proceedings of Bressanone, Scienza e Ben Culturali, XXI. 2005, Sulle pitture murali, Bressanone, Italy, 12-15 July, 2005 pp. $233-240$

[12] T.H. Kolbe, Augmented Videos, Panoramas for Pedestrian Navigation, in: G. Gartner (Ed.), Proceedings of the 2nd Symposium on Location Based Services \& TeleCartography 2004. Geowissenschaftliche Mitteilungen, Schriftenreihe der Studienrichtung Vermessungswesen und Geoinformation, vol. 66, TU Wien, 2004, pp. 45-52.

[13] L. Pecchioli, Accessing Information navigating in a 3D interactive environment, European Doctorate in Technology and Management of Cultural Heritage, XX Cycle, IMT Institute for Advanced Studies, 18 December 2008, Lucca, Italy. 